\title{
Advancing Product Quality: a Summary of the Inaugural FDA/PQRI Conference
}

\author{
Lawrence X. Yu, ${ }^{1,15}$ Jeffrey Baker, ${ }^{1}$ Susan C. Berlam, ${ }^{2}$ Ashley Boam, ${ }^{1}$ E. J. Brandreth,${ }^{3}$ Lucinda Buhse, ${ }^{1}$ \\ Thomas Cosgrove, ${ }_{1}^{1}$ David Doleski, ${ }^{1}$ Lynne Ensor, ${ }^{1}$ Joseph Famulare, ${ }^{4}$ Mohan Ganapathy, ${ }^{7}$ Gustavo Grampp, ${ }^{5}$ \\ David Hussong, ${ }^{1}$ Robert Iser, ${ }^{1}$ Gordon Johnston, ${ }^{5}$ Filippos Kesisoglou, ${ }^{6}$ Mansoor Khan, ${ }^{1}$ Steven Kozlowski, ${ }^{1}$ \\ Emanuela Lacana, ${ }^{1}$ Sau L. Lee, ${ }^{1}$ Stephen Miller, ${ }^{1}$ Sarah Pope Miksinski, ${ }^{1}$ Christine M. V. Moore, ${ }^{1}$ \\ Theresa Mullin, ${ }^{1}$ G. K. Raju, ${ }^{8,9}$ Andre Raw, ${ }^{1}$ Susan Rosencrance, ${ }^{1}$ Mark Rosolowsky, ${ }^{10}$ Paul Stinavage, ${ }^{11}$ \\ Hayden Thomas, ${ }^{12}$ Russell Wesdyk, ${ }^{1}$ Joerg Windisch, ${ }^{13}$ and Sivakumar Vaithiyalingam ${ }^{14}$
}

Received 24 January 2015; accepted 16 March 2015; published online 4 April 2015

\begin{abstract}
On September 16 and 17, 2014, the Food and Drug Administration (FDA) and Product Quality Research Institute (PQRI) inaugurated their Conference on Evolving Product Quality. The Conference is conceived as an annual forum in which scientists from regulatory agencies, industry, and academia may exchange viewpoints and work together to advance pharmaceutical quality. This Conference Summary Report highlights key topics of this conference, including (1) risk-based approaches to pharmaceutical development, manufacturing, regulatory assessment, and post-approval changes; (2) FDA-proposed quality metrics for products, facilities, and quality management systems; (3) performance-based quality assessment and clinically relevant specifications; (4) recent developments and implementation of continuous manufacturing processes, question-based review, and European Medicines Agency (EMA)FDA pilot for Quality-by-Design (QbD) applications; and (5) breakthrough therapies, biosimilars, and international harmonization, focusing on $\mathrm{ICH}$ M7 and Q3D guidelines. The second FDA/PQRI conference on advancing product quality is planned for October 5-7, 2015.
\end{abstract}

\section{INTRODUCTION}

The purpose of the Conference on Evolving Product Quality, under the sponsorship of the Food and Drug Administration (FDA) and Product Quality Research Institute (PQRI), is to bring regulators, industry professionals,

Opinions expressed in this manuscript are those of Lawrence X. Yu, Jeffrey Baker, Ashley Boam, Lucinda Buhse, Thomas Cosgrove, David Doleski, Lynne Ensor, David Hussong, Robert Iser, Mansoor Khan, Steven Kozlowski, Emanuela Lacana, Sau L. Lee, Stephen Miller, Sarah Pope Miksinski, Christine M. V. Moore, Theresa Mullin, Andre Raw, Susan Rosencrance, and Russell Wesdyk and do not necessarily reflect the views or policies of the FDA.

${ }^{1}$ Food and Drug Administration, Center for Drug Evaluation and Research, Silver Spring, Maryland 20993, USA.

${ }^{2}$ Pfizer, Inc, Eastern Point Road, Groton, Connecticut 06340, USA.

${ }^{3}$ Inovio, 660 West Germantown Pike, Suite 110, Plymouth Meeting, Pennsylvania 19462, USA.

${ }^{4}$ Genentech/Roche, 1 DNA Way, South San Francisco, California 94080, USA.

${ }^{5}$ Amgen, One Amgen Center Drive, Thousand Oaks, California 91320, USA.

${ }^{6}$ Generic Pharmaceutical Association (GPhA), 777 Sixth Street, NW, Suite 510, Washington, District of Columbia 20001, USA.

${ }^{7}$ Merck and Company, Inc., West Point, Pennsylvania 19486, USA.

${ }^{8}$ Massachusetts Institute of Technology, Cambridge, Maryland 02139, USA. and academic researchers together and advance pharmaceutical quality. The FDA/PQRI 2014 conference consisted of a plenary session and 12 breakout sessions arranged in four major tracks: risk management and quality metrics; performance-based quality assessment; innovation in manufacturing and regulatory assessment; and emerging
${ }^{9}$ Light Pharm Inc., Cambridge, Maryland 02142, USA.

${ }^{10}$ Bristol-Myers Squibb, 551 Lawrenceville Road, Princeton, New Jersey 08540, USA.

${ }^{11}$ Pfizer Global Manufacturing, 7171 Portage Road, Kalamazoo, Michigan 49001, USA.

${ }^{12}$ Vertex Pharmaceuticals, 50 Northern Avenue, Boston, Maryland 02210, USA.

${ }^{13}$ Sandoz Biopharmaceuticals, 100 College Road West, Princeton, New Jersey 08540, USA.

${ }^{14}$ Teva Pharmaceuticals USA, 223 Quaker Rd, Pomona, New York 10970, USA.

${ }^{15}$ To whom correspondence should be addressed. (e-mail: lawrence.yu@fda.hhs.gov) 
regulatory topics. The following report follows the organization of topics presented at the conference.

\section{ENSURING THE QUALITY OF PHARMACEUTICALS}

In 2004, the FDA published its final report on pharmaceutical quality for the twenty-first century (1), laying out a vision to modernize the regulation of pharmaceutical manufacturing and enhance product quality. As articulated by Janet Woodcock, Director of the FDA Center for Drug Evaluation and Research (CDER), this vision would be realized in "a maximally efficient, agile, flexible manufacturing sector that reliably produces high-quality drug products without extensive regulatory oversight." In the past decade, a number of FDA Guidances have been issued to drive progress toward this vision, in areas including process analytical technology (2), pharmaceutical development (3), quality risk management (4), pharmaceutical quality systems (5), and development and manufacture of drug substances (6). But despite significant progress across the drug development landscape, the American public is facing unprecedented drug shortages and recalls, many of which are related to substandard practices that have undermined pharmaceutical quality. These events have underscored the need for the FDA to reinvigorate its pharmaceutical quality oversight, transform its review and inspection practices, and streamline its regulatory processes.

To satisfy these imperatives, the FDA has worked to create, within CDER, the new Office of Pharmaceutical Quality (OPQ). With a motto of "One Quality Voice," OPQ will ensure a consistent and unified approach to assessing pharmaceutical quality, for stakeholders both internal and external to the agency, and to integrating elements of review, inspection, surveillance, policy, and research. This integrated approach to quality assessment will mobilize CDER expertise, in the form of OPQ teams, who will provide patientfocused, risk-based recommendations. The OPQ surveillance function, driven by a modern informatics platform, will correspondingly determine the status of product quality and facility. The creation of OPQ will concertedly support FDA efforts to assure that all human drugs meet the same quality standards to safeguard clinical performance; to enhance science- and risk-based regulatory approaches; to transform product quality oversight from a qualitative to a quantitative and expertise-based assessment; to integrate review, inspection, surveillance, and research seamlessly across the product lifecycle; and to facilitate the development and adoption of emerging pharmaceutical technology.

The genesis of OPQ has been fueled by recognition that the foundation for consistently high-quality pharmaceuticals is Quality by Design ( $\mathrm{QbD})$. QbD is a systematic approach to development, driven by sound science and quality risk management $(3,7)$ that begins with predefined objectives. QbD encompasses the thorough understanding and optimized control of product and process. The pharmaceutical industry is gradually adopting QbD principles in drug development and commercial manufacturing, and global improvements are accordingly expected, including better capabilities to identify and resolve manufacturing issues quickly and to assess processes continually for improvement opportunities. QbD promises significant benefits to industry and regulators and, more importantly, to consumers.

Efforts to ensure the quality of pharmaceuticals must extend over the course of all manufacturing activities, from product inception through commercialization until discontinuation. This lifecycle approach to product management requires cross-functional teams that provide strategic leadership to product quality. Lifecycle product management proactively integrates product portfolio visibility and oversight, enhances product and process capability, brings rigor to the supply chain, and assesses factors impacting quality as it is implemented throughout the product lifecycle.

QbD and lifecycle product management can emanate from a culture that values continual learning and that expresses continual learning as a principle in its operational governance, employee engagement and enablement, pharmaceutical quality system robustness, and fitness for purpose. Fitness for purpose must be rendered in all aspects of facility, workforce, product mix, process, equipment, technology, and capacity. QbD, lifecycle product management, and continual learning, among other core values, will help to ensure the quality of pharmaceutical products available to the American public.

\section{QUALITY RISK MANAGEMENT AND QUALITY METRICS}

\section{Quality Risk Management for Manufacturing and Inspection}

The comprehensive assessment of risk to quality and to the effective management of quality in pharmaceutical manufacturing is of paramount concern, frequently discussed under the rubric of quality risk management (QRM). Responsibilities to QRM can be ensured through manufacturer self-audits and through regulatory inspections. Both domestic and international perspectives must be considered, focusing on Pharmaceutical Inspection Convention and Pharmaceutical Inspection Co-operation Scheme (http:// www.picscheme.org/).

There are multiple QRM approaches, utilized by both industry and regulators, to ensure product quality and continual improvement $(1,3,4,8,9)$. Clearly, ICH Q9, along with risk management tools derived therefrom, is important with respect to assessing the overall operation of a manufacturing site, the robustness of manufacturing processes, and the quality of the products produced at any given site. Continuing discussion among industrial and regulatory stakeholders will be essential to the development and utilization of QRM methodologies, so that measures to understand risk will remain robust and strategies to mitigate risk will be accordingly applied.

\section{Quality Metrics}

It has become increasingly apparent in recent years that shortages of drugs pose a significant public health threat. The prevention of drug shortages remains a priority concern of the FDA. The FDA has found that most drug shortages stem from quality concerns, either manifested in a finished product or instigated by substandard manufacturing facilities or 
processes that, upon detection, interrupt production and lead to the shortage.

To better prevent drug shortages, the FDA intends to collect quality data and apply quantitative methods to provide metrics that can gauge product quality, provide facility status, and assess manufacturers' quality management system. Quality metrics will be used, in part, to identify establishments at risk for quality deficiencies and supply disruption. Such metrics could support the efficiency of FDA inspections and provide a basis for post-approval regulatory requirements and strategies to ensure product quality.

The FDA has proposed a set of quality metrics for effective surveillance of product quality and facility operations (10). The proposed metrics include lot acceptance rate, right first-time rate, product quality complaint rate, invalidated out-of-specification rate, and annual product review-on-time rate. Metrics of potential interest may include management engagement, use of process capability/performance, and corrective and preventive action rate.

Process capability offers a useful quantitative metric of quality as assessed during drug product design and development, process scale-up and qualification, and routine commercial manufacturing. Because the calculation of the process capability or process capability index $\left(C_{\mathrm{p}}\right.$ and $\left.C_{\mathrm{pk}}\right)$ accounts only for random variability under ideal conditions, the assessed variation is inherent to the process itself (i.e., process noise) (11). When the process has not been demonstrated as stable, it is not process capability, but rather process performance or process performance index $\left(P_{\mathrm{p}}\right.$ or $\left.P_{\mathrm{pk}}\right)$ that is assessed. Thus, $P_{\mathrm{pk}}$ cannot be used as an indicator to forecast the future batch failure rate because it does not describe the process in a state of control. The $P_{\mathrm{pk}}$ value pertains only to process performance as reflected by observed data; a satisfactory $P_{\mathrm{pk}}$ value rating cannot be used to predict performance because the process is not stable. Process capability and performance can be used as indicators of product quality and process stability.

Plans to explore quality metrics and their utility are underway, and parallel activities have been initiated by the International Society for Pharmaceutical Engineering (ISPE) and the Parenteral Drug Association (PDA). The ISPE pilot intends to test a set of quality performance metrics based on industry and FDA input, harmonize a set of metric definitions, test feasibility of data collection across different companies, explore industry practices in quality culture and use of process capability, and share industry input with the FDA.

The PDA survey is intended to develop metrics that can describe the quality culture, which can be defined as an environment in which employees not only follow quality guidelines but also consistently see others taking qualityfocused actions, hear others talking about quality, and "can 'live' quality in all their actions" (12). It is important to recognize that financial incentives alone cannot motivate employees to embrace such a culture. The PDA survey intends to help identify attributes of quality culture and quality system maturity, correlate quality culture behavior scoring (subjective) to quality system maturity scoring (objective), and determine whether quality system maturity scoring can differentiate sites.

\section{PERFORMANCE-BASED QUALITY ASSESSMENT}

\section{Clinically Relevant Specifications}

To ensure that drugs available to patients are consistently safe and effective, quality assessment must incorporate clinically relevant specifications (CRSs). Robust specifications linked to clinical outcomes will ensure that drug products are equivalent, in clinically meaningful terms, from one lot to the next as well as between innovator and generic products.

An example of incorporating clinical relevance into product quality can be seen in the increased attention being focused upon dissolution as a surrogate for bioequivalence. Biopharmaceutics Classification System (BCS) subclasses, as proposed by Dr. Gordon Amidon and shown in Table I, can inform development of predictive dissolution tools $(13,14)$. A structured five-step approach can be envisaged as starting with a quality risk assessment, followed by development of tests for critical quality attributes (CQAs), followed by testing for impact of CQAs in the clinic, and finally using the data to establish CQA limits for incorporation into subsequent QbD steps. Challenges to implementing a CRS approach include the development of functional models and the availability of data at the time of product approval.

Simulated case studies that allow dissolution data to be compared with bioequivalence outcomes highlight the aforementioned challenges. Dissolution methodologies can include bio-relevant media and conditions (e.g., non-sink methodologies) and accompany continuous investment in in vitro and in vivo correlation (IVIVC), especially for modified-release products. An initial focus on low-risk cases may illustrate the benefits of CRSs. Table I presents dissolution standards under various BCS subclasses. Development of scientific tools will benefit from close communication between industry and regulatory agencies to ensure mutual understanding of data and expectations.

\section{Lifecycle Management and Post-approval Changes (Current Practice-Future Directions)}

Global emphasis on the performance of the industry and lifecycle management of drug products must extend to postapproval. Important and interrelated aspects of this emphasis are (1) management of post-approval changes to chemistry, manufacturing, and controls (CMC) following risk- and science-based approaches; (2) the integration of production data (e.g., batch data) into the current regulatory framework (e.g., annual reports); and (3) product quality assurance and performance-based regulation principles to prevent drug shortages.

Health Canada recently downregulated certain postapproval changes that had previously required a level I supplement (i.e., description of a major quality change) (15). Canada now has two reporting categories; one pertains to approved supplements before implementing a change, and the other is satisfied through annual notification. It was believed that the changes in regulatory standards in Canada, whereby certain changes in product or process parameters for drug substances and drug products are now allowed via annual notification, reflect the use of modern principles of 
Table I. BCS Subclassification and Dissolution Testing for Immediate-Release Dosage Forms

\begin{tabular}{|c|c|c|c|c|}
\hline BCS subclassification & $\begin{array}{l}\text { Solubility at } \\
\text { pH } 2\end{array}$ & $\begin{array}{l}\text { Solubility at } \\
\text { pH } 6.8\end{array}$ & Permeability & Dissolution testing \\
\hline I & High & High & High & $>85 \%$ dissolution in $15 \mathrm{~min} ; 30 \mathrm{~min}, \mathrm{~F} 2>50, \mathrm{pH}=6.8$ \\
\hline IIA $^{a}$ & Low & High & High & $\begin{array}{l}15 \text { min at } \mathrm{pH}=1.2 \text {, then } 85 \% \text { dissolution in } 30 \text { min, } \mathrm{pH}=6.8 \text {; } \\
\mathrm{F} 2>50 ; 5 \text { points minimum, not more than } 1 \text { point }>85 \%\end{array}$ \\
\hline $\operatorname{IIB}^{b}$ & High & Low & High & $>85 \%$ dissolution in $15 \mathrm{~min}, \mathrm{pH}=1.2$ \\
\hline $\mathrm{IIC}^{c}$ & Low & Low & High & $\begin{array}{l}15 \text { min at } \mathrm{pH}=1.2 ; \text { then } 85 \% \text { dissolution in } 30 \mathrm{~min}, \mathrm{pH}=6.8 \\
\text { plus surfactant } \mathrm{t}^{a} ; \mathrm{F} 2>50 ; 5 \text { points minimum, not more than } \\
1 \text { point }>85 \%\end{array}$ \\
\hline III & High & High & Low & $>85 \%$ dissolution in $15 \mathrm{~min}, \mathrm{pH}=1.2,4.5,6.8$ \\
\hline $\mathrm{IVA}^{a}$ & Low & High & Low & $\begin{array}{l}15 \mathrm{~min} \text { at } \mathrm{pH}=1.2 \text {; then } 85 \% \text { dissolution in } 30 \mathrm{~min}, \mathrm{pH}=6.8 \text {; } \\
\mathrm{F} 2>50 ; 5 \text { points minimum, not more than } 1 \text { point }>85 \%\end{array}$ \\
\hline $\operatorname{IVB}^{b}$ & High & Low & Low & $>85 \%$ dissolution in $15 \mathrm{~min}, \mathrm{pH}=1.2$ \\
\hline $\mathrm{IVC}^{c}$ & Low & Low & Low & $\begin{array}{l}15 \text { min at } \mathrm{pH}=1.2 ; \text { then } 85 \% \text { dissolution in } 30 \mathrm{~min}, \mathrm{pH}=6.8 \\
\text { plus surfactant } \mathrm{t}^{a} \mathrm{~F} 2>50 ; 5 \text { points minimum, not more } \\
\text { than } 1 \text { point }>85 \%\end{array}$ \\
\hline
\end{tabular}

${ }^{a}$ With a $\mathrm{pKa}<5$

${ }^{b}$ With a $\mathrm{pKa}>6$

${ }^{c}$ With no PK(s) or $\mathrm{pKa}(\mathrm{s})<0$ or $>8$

quality risk management and incorporation of key concepts found in ICH Q8, Q9, and Q10.

Lifecycle management encompasses statutes, regulations, and FDA guidance related to post-approval changes. The limitations of the current regulatory approaches on postapproval changes include (1) risk management concepts are applied, but often under the assumption that all products within a drug product category have similar risk (i.e., onesize-fits-all approach); (2) regulatory approaches are often too conservative and occasionally overly liberal; and (3) there is insufficient flexibility to recognize well-understood risks to product quality or manufacturing processes. Hence, welldefined established conditions or regulatory commitments could allow for more effective post-approval change management and promote innovation and continual improvement. This would also provide FDA with pathways to better regulate post-approval changes by utilizing more flexibility and science- and risk-based principles.

According to the Pareto principle, attention to $20 \%$ of the highest risk areas of production could mitigate the great majority of product quality failure (16). According to cGMP regulations, drug product manufacturers must assess all changes under their pharmaceutical quality systems (PQS); however, only the changes to the high-risk areas need to be reported to FDA, and low-risk changes could be managed solely by an applicant's PQS. Even though many challenges still exist from a scientific, regulatory, and PQS perspective for communication and management, clearly defined established conditions or regulatory commitments could lead to a considerable reduction in the regulatory burden related to post-approval changes.

\section{INNOVATION IN MANUFACTURING AND ASSE SSMENT}

\section{Continuous Manufacturing}

Continuous processing has the potential to reduce the costs of pharmaceutical manufacturing processes and to improve product quality. Over the past decade, significant investments have been made in the development of continuous processes by the pharmaceutical industry, equipment manufacturers, academia, and government agencies. These investments have resulted in new enabling manufacturing technologies (e.g., continuous crystallization) and advances in process models, process analytical technology (PAT) tools, and advanced process control strategies. These advances, along with the adoption of the QbD paradigm for pharmaceutical development, represent new levels of scientific and regulatory readiness for continuous manufacturing.

Continuous manufacturing provides the opportunity for process integration, including end-to-end integration of drug substance and drug product manufacturing. The novel concept of an end-to-end continuous manufacturing platform has been applied to continuous crystallization as an enabling technology for production of active pharmaceutical ingredients (APIs) (17). Continuous crystallization provides built-in flexibility for control that may lead to new approaches to product quality control. The control of polymorphism in a continuous crystallization process offers an example of the effective manipulation of process parameters in a continuous manufacturing context.

Continuous manufacturing may offer avenues to accelerated development which may be ideal for breakthrough therapies. Continuous and fast responses to any changes in process parameters allow more information to be gathered from smaller quantities of API, so that data-rich process models may be developed. Predictions from combined process models can be confirmed with fully continuous process experiments streamlining QbD development.

Continuous manufacturing offers the opportunity to develop and better utilize process models because the complexity of the governing equations may be reduced compared to batch processes. Several model-based approaches for design and control of continuous crystallizers for manufacturing APIs have been described, for example, to predict how temperature variation and processing time of each stage may affect purity and yield $(18,19)$. Flowsheet 
models, which represent a sequence of unit operations, can be applied to pharmaceutical processes in order to conduct preliminary design evaluation, identify potential bottlenecks, compare control strategies, identify potential critical process parameters, explore design space, and enhance process understanding (20). Dynamic modeling can be used to understand traceability and the propagation of variability in continuous systems. In addition, flowsheet models can provide insights into dynamic behavior of pharmaceutical processes (e.g., direct compression, wet granulation, etc.). Flowsheet model simulations can also be used to attribute potential variability in product quality to sources of variability in the process, thereby facilitating the risk assessment of processes and control strategies.

Process analytical technology (PAT) becomes critical for the monitoring and control of continuous processes, due to the absence of isolated intermediates. Similarly, process dynamics for a continuous process are typically faster, and thereby may call for more frequent measurements provided by PAT. Real-time data provided by PAT systems enable the implementation of advanced process control (APC) systems, which are devised, based on knowledge of process dynamics, to reduce the frequency, severity, and costs of transient excursions. An APC system for a blending process, for example, may utilize a combination of feedback (e.g., measured deviations from the desired quality target) and feed-forward approaches (e.g., preemptive adjustments that anticipate feeder excursions and maintain blend uniformity). APC is a key enabling technology for continuous processing. In addition to supporting the implementation of APC, exploiting process and quality data collected during continuous production facilitates the adoption of multivariate statistical process control (MSPC) for process monitoring. MSPC approaches support proactive interventions into process disturbances that might otherwise result in out-ofspecification products, equipment malfunctions, or process safety incidents.

\section{Innovations in Manufacturing and Regulation for Sterile Drug Products}

A variety of challenges currently confront sterile product manufacturing, and numerous companies have accordingly begun to pursue new manufacturing paradigms and advanced technologies in microbiological detection, environmental monitoring and control, aseptic processing, and terminal sterilization. Traditional testing and monitoring methods in microbiology often require significant resources, which can be time-consuming, labor-intensive, and costly. However, the implementation of new technology to assist in this monitoring and/or testing may ultimately allow for greater regulatory flexibility, improved process and product knowledge, and faster manufacturing adaptability.

The implementation of any new manufacturing technology must be based on a thorough knowledge of the product's critical quality attributes. Equipped with this knowledge, the exploration into effective ways to incorporate innovative technology into the manufacturing process may be initiated, with QbD principles as a foundation. Consideration of all product attributes (e.g., source materials, container closure system, route of administration, product dosing, product labeling, and storage conditions) is essential to the development of sterile drug products as well as the development of any relevant new technology. Environmental monitoring programs, at both the facility and filling-line level, must also be considered, along with manufacturing process and product release and stability specifications.

\section{Question-based Review and the Future of Regulatory Submissions}

The Question-based Review (QbR) framework, utilized in CDER and CVM integrates important scientific and regulatory review questions into regulatory submissions. The QbR framework facilitates the communication of risk assessment activities, engenders a comprehensive description of product and process development, and envisages an overall control strategy for drug products assessed by the FDA. Industry professionals and regulators benefit reciprocally from the risk-based evaluation of applications and the integration of risk management into development, communication, and management plans.

QbR questions, which can be integrated into the Quality Overall Summary (QOS), should be asked and addressed internally, in real time, during pharmaceutical development activities, rather than writing answers after the fact for the purposes of submission. QbR creates a framework for the applicant to provide a concise knowledge base for review and lifecycle activities, as opposed to the detail-rich Module 3 of the Common Technical Document (CTD). The QbR framework, moreover, incorporates $\mathrm{QbD}$ principles.

QbR questions are meant to be flexible; irrelevant questions may be omitted and related questions may be grouped together to provide a concise overview. The FDA has taken efforts to ensure that quality assessments using QbR should not exclude critical information of the submission. A CDER MaPP on QbR has been issued (21).

\section{EMERGING REGULATORY TOPICS}

\section{Breakthrough Therapy for Large and Small Molecules}

The breakthrough pathway, introduced under the FDA Safety and Innovation Act of 2012, is designed to expedite the development of drugs for life-threatening conditions or unmet medical needs (22). The program provides intensive FDA guidance on efficient drug development, involvement of senior FDA management in the review process, and a crossdisciplinary project lead that ensures efficient communication between FDA and applicants. The breakthrough designation is intended to signal a potentially transformative therapy, characterized, for example, by markedly higher response rates over available therapies.

The breakthrough pathway poses challenges to the FDA, particularly in terms of resources, as well as to applicants seeking breakthrough designations and the eventual marketing of breakthrough products. Even as the FDA strives to bring flexibility to its expedited review programs, reviewers must adhere to proven standards of efficacy, safety, and quality. For industry, the breakthrough pathway challenges sponsors to communicate process and product information, preliminary information on the appropriate formulation, 
specifications based on limited numbers of lots, and sufficient stability data congruent with a commercially viable expiration protocol. Site inspection readiness constitutes an additional and significant challenge. Additional challenges posed by an accelerated development program are logistical and relate to the potential need for larger amounts of product for clinical development and for launching the products in multiple, global markets. Potential solutions to these problems may include risk-based prioritization of process and method development; deferral of non-critical CMC activities to the post-launch stage (e.g., process linkage studies); provisional specification, whereby a consistent product is initially sought that can be refined as knowledge is acquired; the use of comparability protocols or Post Approval Lifecycle Management Plans; and product launch from clinical sites with reliance on the compliance history of manufacturing sites.

The FDA is regularly charged with weighing inherent levels of risk against the potential for clinical benefit in patients, and benefit-risk analyses will be inherent in quality assessments. The agency understands the concerns identified by industry related to process and product understanding, lack of stability data and preliminary understanding of formulation and CQAs, and scale-up and manufacturing site readiness. To alleviate some of the concerns, FDA uses novel approaches to the review of breakthrough drugs, including discussion of risk mitigation strategies to manage risk to product quality, the possibility of streamlining the review of stability data, use of post-marketing requirements and postmarketing commitments to address post-approval risk, and proactive communication between review and inspection staff during assessment of applications. Proactive and risk-based communication is clearly essential to the success of the breakthrough program.

\section{Biosimilars: the Finish Line and Beyond}

The demonstration of a product as a biosimilar, based on structural and functional similarity with its reference product, encompasses particular quality concerns. As we look forward to a time when multiple versions of a given biologic may be approved, we must anticipate the tools that will be needed to support the manufacture and marketing of high-quality biosimilars. It is clear that FDA is encouraging innovation in the pursuit of fingerprint-like similarity to reduce residual uncertainty, calling for better analytical tools and pattern analyses (23). Residual uncertainty owing to differences in potentially critical physicochemical attributes is a key challenge in biosimilar development, and sponsors may avail themselves of sophisticated, orthogonal functional and nonclinical studies to address such uncertainty. Given the limited opportunity to precisely determine a reference product's population statistics (e.g., owing to limited availability of independent lots or due to reference product drift over time), there is no ideal statistical methodology for similarity that can cover all relevant quality attributes. When there is reduced statistical confidence in a similarity comparison (e.g., due to limited numbers of lots), post-approval control strategies may have to become more stringent.

Modern QbD and pharmaceutical quality systems promise to limit drift or divergence in CQAs among biologics, and any intended evolution in CQAs of a given biologic should preserve the bridge to those datasets that had been used in making approval decisions. There are no special burdens in this regard for biosimilar sponsors. Finally, there may be a role for public standards in assisting sponsors with method performance qualification and with benchmarking potency or purity methods and attributes against physical standards. However, public standards (e.g., product-specific monographs) should only be developed if they empower regulators and sponsors and address an unmet need. In the coming years, the biosimilar regulatory pathway will have to recognize these many challenges and potential solutions and manage new knowledge as it evolves from case studies related to $\mathrm{CMC}$ issues.

\section{International Harmonization}

Harmonization activities remain crucial in our global environment. Currently, there are numerous CMC requirements around the globe that are applied in the review of new applications as well as in post-approval submissions. But any incongruity in CMC requirements may result in staggered health authority review/approval times, resulting in inventory fragmentation and drug shortage risks. In addition, health authority inspections are often redundant, which unnecessarily drains resources of both industry and regulatory agencies. For example, it may take up to 4 years to get one postapproval change approved globally.

One recent harmonization guideline that aspires to provide a common data package for global submission and approval of regulatory applications is the ICH M7 Guideline on Assessment and Control of DNA Reactive (Mutagenic) Impurities in Pharmaceuticals to Limit Potential Carcinogenic Risk (24). The M7 guideline addresses new drug substances and new drug products during their clinical development and subsequent applications for marketing. It also applies to postapproval submissions of marketed products and to new marketing applications for products with a drug substance that is present in an approved product.

M7 provides recommendations for assessing and eliminating mutagenic impurities in drug substances or drug products in a risk-based manner. The guideline encompasses structure-activity relationships to assess and predict mutagenicity potential (i.e., hazard identification) and determination of risk (i.e., risk assessment). It applies the concept of Threshold of Toxicological Concern (TTC) and classifies impurities into five classes based on mutagenicity and carcinogenicity. It uses less-than-lifetime (LTL) limits based on duration of use, providing a flexible and practical approach during clinical development and marketing. M7 outlines flexible ways to control mutagenic impurities and a staged approach to documentation during development.

The ICH Q3D guideline provides guidance on elemental impurities, establishing permitted daily exposure based on acceptable safety limits of potentially toxic elemental impurities. This guideline applies to new finished drug products and new drug products employing existing drug substances. Drug products containing proteins and polypeptides, their derivatives, and products of which they are components (e.g., conjugates) are in the scope of this guideline, as are drug products containing synthetically produced polypeptides, polynucleotides, and oligosaccharides. The primary content of the ICH Q3D guideline was 
finalized in June 2014, and the ICH Q3D expert working group is charged to establish an approach to evaluating large-volume parenteral products, including enteral nutrition, and elaborate a harmonized procedure on application of the guideline to currently marketed products.

There is limited data in the open literature concerning elemental impurities in commonly used drug product components, and implementation of the ICH Q3D guideline will provide valuable information. Trade organizations are initiating collaborative work to develop and share additional information; harmonization of document compliance with the requirements of $\mathrm{ICH}$ Q3D in regulatory submissions will be helpful. The implementation of ICH Q3D provides an opportunity to put into practice a risk- and science-based approach to the control of elemental impurities. The real risks of elemental impurity inclusion in drug products will become clearer, providing an ability to focus efforts judiciously.

The EMA and FDA have initiated a pilot for QbD applications. The objectives of the pilot are to allow US and EU reviewer/assessors to exchange their views on the implementation of ICH Q8 as they encounter actual applications. The scope of this pilot includes submissions that use at least one of the following: design space, PAT tools for control, continuous process verification, models to support real-time release testing, continuous manufacturing processes, or postapproval regulatory flexibility. The pilot has made significant progress, establishing a platform for continuous dialogue between the agencies. Process improvements to facilitate interactions with industry during the pilot process are already in place for future applications. The pilot appears to promise a number of benefits, including

- Consistent interpretation of the ICH guidelines. The pilot has resulted in three question-and-answer documents, clarifying QbD topics and current regulatory perspectives;

- Joint review and assessment of QbD filings, which will reduce overall cost and resource requirements (for industry) and can potentially lead to harmonization of queries from regulatory authorities and thus streamline the overall approval process; and

- Opportunities for joint FDA-EMA meetings that will streamline regulatory assessment. There is a potential for joint inspection of QbD applications to reduce the inspection burden and increase reviewer-inspector interactions across agencies.

\section{SUMMARY}

The first FDA/PQRI conference discussed numerous, important developments of regulatory policies on product quality, which include

- Science- and risk-based approaches to development, manufacturing, regulatory assessment, and inspection;

- FDA-proposed metrics of drug products, facilities, and quality management systems;

- Development and utility of clinically relevant dissolution specifications;

- Perspectives on future regulatory submissions including question-based review and communication of regulatory commitments, noting key lessons from the EMA-FDA pilot for QbD applications; and

- Current thoughts on emerging areas such as continuous manufacturing, breakthrough therapies, biosimilars, and international harmonization of mutagenic and elemental impurity guidelines.

The second annual FDA/PQRI Conference on Advancing Product Quality is planned for October 5-7, 2015.

\section{REFERENCES}

1. U. S. Food and Drug Administration. Final report on pharmaceutical cGMPs for the 21st century-a risk-based approach. 2004.

2. U. S. Food and Drug Administration. Guidance for industry: PAT - a framework for innovative pharmaceutical development, manufacturing, and quality assurance. 2004.

3. U. S. Food and Drug Administration. Guidance for industry: Q8(2) pharmaceutical development. 2009.

4. U. S. Food and Drug Administration. Guidance for industry: Q9 quality risk management. 2006.

5. U. S. Food and Drug Administration. Guidance for industry: Q10 pharmaceutical quality system. 2009.

6. U. S. Food and Drug Administration. Guidance for industry: Q11 development and manufacture of drug substances. 2012.

7. Yu LX, Amidon G, Khan MA, Hoag SW, Polli J, Raju GK, et al. Understanding pharmaceutical quality by design. AAPS J. 2014;16:771-83.

8. Frank T, Brooks S, Creekmore R, Hasselbalch B, Murray K, Obeng K, et al. Quality risk management principles and industry case studies. The Pharmaceutical Quality Research Institute Manufacturing Technology Committee. 2008. http://pqri.org/pdfs/MTC/ Quality_Risk_Management_Principles_and_Industry_Case_Studies_December_28_2008.pdf. Accessed 02 Mar 2015.

9. Potter C. PQLI application of science-and risk-based approaches (ICH Q8, Q9, and Q10) to existing products. J Pharm Innov. 2009;4(1):4-23.

10. Measuring pharmaceutical quality through manufacturing metrics and risked-based assessment. 2014. http:// www.brookings.edu/ /media/research/files/papers/2014/05/01quality-metrics-fda/quality-metrics-meeting-summary.pdf. Accessed 02 Mar 2015.

11. Pearn WL, Lin PC. Testing process performance based on capability index $\mathrm{C}_{\mathrm{pk}}$ with critical values. Comput Ind Eng. 2004;47(4):351-69.

12. Srinivasan A, Kurey B. Creating a culture of quality. Harv Bus Rev. 2014;92(4):23-5.

13. Tsume Y, Mudie DM, Langguth P, Amidon GE, Amidon GL. The Biopharmaceutics Classification System: subclasses for in vivo predictive dissolution (IPD) methodology and IVIVC. Eur J Pharm Sci. 2014;57:152-63.

14. Amidon GL, Lennernäs H, Shah VP, Crison JR. A theoretical basis for a biopharmaceutic drug classification: the correlation of in vitro drug product dissolution and in vivo bioavailability. Pharm Res. 1995;12(3):413-20.

15. Health Canada is pleased to announce the release of the revised Post-Notice of Compliance (NOC) Changes-quality guidance. 2013. http://www.hc-sc.gc.ca/dhp-mps/alt_formats/pdf/ prodpharma/applic-demande/guide-ld/postnoc_change_apresac/ noc_pn_quality_ac_sa_qualite-eng.pdf. Accessed 02 Mar 2015.

16. Sanders R. The Pareto principle: its use and abuse. J Serv Mark. 1987;1(2):37-40.

17. Cervera-Padrell AE, Skovby T, Kiil S, Gani R, Gernaey KV. Active pharmaceutical ingredient (API) production involving continuous processes - a process system engineering (PSE)assisted design framework. Eur J Pharm Biopharm. 2012;82(2):437-56.

18. Alvarez AJ, Myerson AS. Continuous plug flow crystallization of pharmaceutical compounds. Cryst Growth Des. 2010;10(5):221928. 
19. Nagy ZK, Braatz RD. Advances and new directions in crystallization control. An Rev Chem Biomol Eng. 2012;3:55-75.

20. Sen M, Chaudhury A, Singh R, John J, Ramachandran R. Multiscale flowsheet simulation of an integrated continuous purification-downstream pharmaceutical manufacturing process. Int $\mathbf{J}$ Pharm. 2013;445(1):29-38.

21. FDA Center for Drug Evaluation and Research (CDER) issued a QbR MAPP for chemistry review. 2014. http://www.fda.gov/downloads/ AboutFDA/CentersOffices/OfficeofMedicalProductsandTobacco/ CDER/ManualofPoliciesProcedures/UCM423752.pdf. Accessed 06 Dec 2014

22. Food and Drug Administration Safety and Innovation Act (FDASIA).2012. http://www.fda.gov/RegulatoryInformation/
Legislation/FederalFoodDrugandCosmeticActFDCAct/ Significant A mendmentstotheFDCAct/FD A S I A/ ucm20027187.htm.

23. U. S. Food and Drug Administration. Guidance for industry: quality considerations in demonstrating biosimilarity to a reference protein product. 2012. http://www.fda.gov/downloads/ Drugs/GuidanceComplianceRegulatoryInformation/Guidances/ UCM291134.pdf. Accessed 28 Feb 2015.

24. International Conference on Harmonization of Technical Requirements for Registration of Pharmaceuticals for Human Use. ICH M7: assessment and control of DNA reactive (mutagenic) impurities in pharmaceuticals to limit potential carcinogenic risk. 2014. 